\title{
CORRELAÇÃO DIAGNÓSTICA ANATOMOCLÍNICA. AFERIÇÃO RETROSPECTIVA DO DIAGNÓSTICO CLÍNICO EM NECRÓPŞIAS*
}

\author{
Marcos Célio de Almeida** \\ Luiz Antonio Andrade Machado Couto*** \\ Luiz Henrique Fernandes da Silva*** \\ Silvio dos Santos Carvalhal***
}

\begin{abstract}
ALMEIDA, M.C. de et al. Correlação diagnóstica anatomoclínica. Aferição retrospectiva do diagnóstico clínico em necrópsias. Rev. Saúde públ., S. Paulo, 23:285-91, 1989.

RESUMO: Analisaram-se retrospectivamente 200 necrópsias, correlacionando-se os diagnósticos clinicos e anatomopatológicos. Foram consideradas 20 entidades nosológicas para confronto. Os diagnósticos não formulados em vida, embora observados na necrópsia (falso negativo), e que apresentaram os maiores percentuais em relação aos respectivos totais de achados necroscópicos, foram: pielonefrite aguda $(100 \%)$, embolia pulmonar $(87,50 \%)$, aneurisma dissecante da aorta $(66,66 \%)$ e broncopneumonia $(58,16 \%)$. Os diagnósticos formulados em vida que não tiveram confirmação pela necrópsia (falso positivo), e que apresentaram os maiores percentuais em relação aos respectivos totais dos diagnósticos clínicos, foram: tuberculose $(69,50 \%)$, paracoccidioidomicose $(57,14 \%)$, septicemia $(53,13 \%)$ e doença de Chagas $(44,44 \%)$. Houve concordância diagnóstica em $97(48,50 \%)$ dos 200 casos. Em $19(9,50 \%)$ deles o desacordo diagnóstico, se antes verificado, poderia ter implicado alteraçðes do prognóstico. Enfatiza-se a importância da necrópsia para uma adequada correlaçåo anatomoclinica e discute-se os achados em relação a estudos prévios.
\end{abstract}

DESCRITORES: Diagnóstico. Autópsia. Erros de diagnóstico. Avaliação.

\section{INTRODUÇÃO}

A necrópsia permite a clínicos e patologistas avaliar, por método direto, os diagnósticos formulados em vida, relacionar os testes clínicos e laboratoriais com os achados anatômicos e avaliar o grau de sucesso do emprego de técnicas cirúrgicas e da utilização de drogas ${ }^{13,33}$.

É evidente que o termo "diagnóstico" pode comportar diferentes graus de probabilidade quanto a estar ou não correto, embora tal graduação não seja feita rotineiramente ${ }^{15}$. Tem-se demonstrado, no entanto, que mesmo quando essa certeza possa existir, a necrópsia leva a alterações significativas nos diagnósticos anteriormente formulados ${ }^{8,10,23,28}$.

Nos últimos anos, tem-se observado acentuada e progressiva queda do número de necrópsias que não sejam de interesse médico-legal ${ }^{40}$. Numerosos trabalhos vieram teafirmar a importância da necrópsia como meio de aferição do dignóstico clínico $5,8,10,13,14,19,20,21,23,26,28,30,32,33,35$, 37,38,42. Como possíveis causas dessa queda tem. se apontado a excessiva confiança nos diagnósticos obtidos pelos sofisticados equipamentos e técnicas laboratoriais, atualmente disponí- veis $8,10,13,20,23$. Outras causas observadas são: a dificuldade de obter-se autorização junto à família, custos elevados e a não implantação sistemática da necrópsia obrigatória, mesmo nos meios universitários $* * * *$.

Nos últimos dez anos, temos observado queda de $40 \%$ no número de necrópsias em relação ao número total de óbitos hospitalares****. Em vista disso, e do fato de não ter sido encontrado trabalho semelhante em publicação nacional, foi realizado o presente estudo em que se correlacionam os diagnósticos clínicos e os diagnósticos necroscópicos.

\section{MATERIAL E MÉTODO}

Realizou-se estudo retrospectivo de correlação diagnóstica anatomóclínica a partir de casos de necrópsias realizadas no Departamento de Anatomia Patológica da Faculdade de Ciências Médicas da Universidade Estadual de Campinas (FCM-UNICAMP), durante os anos de 1980 a 1982. O Hospital da FCM-UNICAMP contava, nesse período, com 280 leitos, laboratório de análises clínicas e de anatomopatologia

* Apresentado no 1 Congresso Médico Universitário do Estado de São Paulo, São Paulo, 1987.

** Departamento de Medicina Complementar da Faculdade de Ciências da Saúde da Universidade de Brasília - 70910 Brasília, DF - Brasil.

*** Departamento de Clínica Médica da Faculdade de Ciências Médicas da Universidade Estadual de Campinas (UNICAMP) - 13100 - Campinas, SP - Brasil.

**** Dados observados rotineiramente pelos autores do presente trabalho - não publicados. 
e serviço de radiodiagnóstico, sem no entanto contar com exames de maior sofisticação (por exemplo: tomografia computadorizada) e tendo serviço restrito de ultrassonografia.

Foram excluídos do estudo recém-nascidos vivos de até uma semana de vida. De um total restante de 400 casos de necrópsia foram analisadas 200 em que o prontuário clínico permitiu a obtenção dos dados utilizados no estudo (cor, sexo, idade, período de internação e hipóteses dianósticas), bem como informações necessárias para a avaliação do diagnóstico e prognóstico (exame de laboratório e terapêutica).

Os dados clínicos registrados foram extraídos da ficha de requisição de necrópsia e do prontuário clínico. Como dados anatomopatológicos, foram utilizadas as descrições macroscópicas e de microscopia óptica da necrópsia.

As hipóteses diagnósticas clínicas foram consideradas formuladas desde que presentes na ficha de requisição de necrópsia ou no prontuário clínico. Uma hipótese clínica era também considerada como tendo sido formulada, mesmo quando não anotada, nem no prontuário clínico nem na ficha de requisição de necrópsia se a terapêutica empregada fosse suficientemente sugestiva de sua formulação (Exemplo: esquema tríplice para tuberculose ou associação de vasodilatadores e diuréticos para hipertensão arterial).

Foram analisados 15 diagnósticos, selecionados dentre trabalhos semelhantes já publicados na literatura, devido à sua grande importância clínico-epidemiológica (risco à vida, prevalência e outros) (Tabela). Foram ainda analisados, no presente estudo, três diagnósticos de importância regional (doença de Chagas, paracoccidioidomicose e neurocisticercose) e diagnósticos que não têm sido freqüentemente relatados neste tipo de estudo (insuficiência cardíaca congestiva e malformações congênitas). Dezessete deles tinham critérios anatomopatológicos bem definidos e já descritos ${ }^{17}$. Para o presente estudo, apresentamos os critérios anatomopatológicos referentes aos diagnósticos de:

a) insuficiência cardíaca congestiva: dilatação de câmaras cardíacas, com ou sem hipertrofia, acompanhada de congestão passiva do fígado;

TABELA

Distribuição dos diagnósticos clínicos e anatomopatológicos segundo as entidades nosológicas. *

\begin{tabular}{|c|c|c|c|c|c|c|c|}
\hline \multirow{2}{*}{ Entidades Nosológicas } & \multirow{2}{*}{ DC } & \multirow{2}{*}{$F(+)$} & \multirow{2}{*}{ DAP } & \multirow{2}{*}{$F(-)$} & \multirow{2}{*}{$\frac{F(-)}{D A P} \times 100$} & \multirow{2}{*}{$\frac{\mathrm{DC}-(\mathrm{F}(+))}{\mathrm{DC}}$} & \multirow{2}{*}{$\frac{F(+)}{D C} \times 100$} \\
\hline & & & & & & & \\
\hline 1. Infarto do miocárdio & 6 & 1 & 7 & 2 & 28,57 & 83,33 & 16,66 \\
\hline 2. Acidente vascular cerebral & 25 & 4 & 26 & 5 & 19,23 & 84 & 16 \\
\hline 3. Pielonefrite aguda & 0 & 0 & 6 & 6 & 100 & 0 & 0 \\
\hline 4. Meningite purulenta & 3 & 0 & 5 & 2 & 40 & 100 & 0 \\
\hline 5. Tuberculose & 23 & 16 & 13 & 6 & 46,15 & 30,43 & 69,56 \\
\hline 6. Embolia e/ou inf. pulmonar & 2 & 1 & 8 & 7 & 87,50 & 50 & 50 \\
\hline 7. Insuf. cardíaca congestiva & 29 & 5 & 35 & 11 & 31,43 & 82,75 & 17,24 \\
\hline 8. Doenca de Chagas & 9 & 4 & 5 & 0 & $\begin{array}{r}J 1,+3 \\
0\end{array}$ & 55,55 & $\begin{array}{l}11,24 \\
44,44\end{array}$ \\
\hline 9. Peritonite & 6 & 0 & 14 & 8 & 57,14 & 100 & $\begin{array}{r}77,77 \\
0\end{array}$ \\
\hline 10. Cirrose hepática & 13 & 5 & 11 & 3 & 27,27 & 61,53 & 38,46 \\
\hline 11. Hemorragia ou infarto T.G.I. & 17 & 7 & 11 & 1 & 9,09 & 58,82 & 41,18 \\
\hline 12. Aneurisma dissecante (a or ta) & 1 & 0 & 3 & 2 & 66,66 & 100 & 0 \\
\hline 13. Neoplasia malígna & 38 & 4 & 45 & 11 & 24,44 & 89,47 & 10,53 \\
\hline 14. Malformação congênita & 8 & 2 & 8 & 2 & 25 & 75 & 25 \\
\hline 15. Broncopneumonia & 49 & 8 & 98 & 57 & 58,16 & 83,67 & 16,33 \\
\hline 16. Hipertensão arterial malígna & 4 & 0 & 4 & 0 & 0 & 100 & 0 \\
\hline 17. Diabetes & 11 & 5 & 7 & 1 & 14,28 & 54,54 & 45,45 \\
\hline 18. Septicemia & 32 & 17 & 19 & 4 & 21,05 & 46,87 & 53,13 \\
\hline 19. Neurocisticercose & 3 & 0 & 6 & 3 & 50 & 100 & 0 \\
\hline 20. Paracoccidioidomicose & 7 & 4 & 4 & 1 & 25 & 42,85 & 57,14 \\
\hline
\end{tabular}

* Número de casos: 170

DC: Diagnóstico clínico; DAP: Diagnóstico anatomopatológico; $F(+)$ : Falso positivo (diagnóstico clínico sem confirmação anatomopatológica); $F(-)$ : Falso negativo (diagnóstico não observado pela clínica, somente relatado na necrópsia); $\frac{F(-)}{\text { DAP }}:$ Estimativa dos diagnósticos anatomopatológicos não observados pela clínica; $\frac{D C-(F(+))}{\mathrm{FC}(+)}:$ Estimativa da confirmação anatomopa tológica do diagnóstico clínico; $\frac{\dot{D C}}{\mathrm{DC}}:$ Estimativa dos diagnósticos clínicos que não encontraram confirmação ana tomopatológica; T.G.I.: Trato gastrointestinal. 
b) diabetes pelo achado da glomerulosclerose intercapilar de Kimmenstiel Wilson, e c) septicemia pelo achado de dois ou mais focos de infecção bacteriana.

Os diagnósticos clínicos e anatomopatológicos foram comparados notando-se a concordância ou a discordância, sendo a primeira dividida em: a) presença de diagnóstico anatomopatológico sem suspeita clínica e b) diagnósticos clínicos sem confirmação anatomopatológica (Tabela).

O erro diagnóstico foi também classificado por sua possível implicação prognóstica levando em consideração os recursos hospitalares disponíveis tanto para diagnóstico como para tratamento, assim como as condutas seguidas ou não. Como exemplo: paciente internado com história de febre vespertina e perda de peso, que após exame físico e radiografia de tórax foi submetido a tratamento com esquema tríplice para tuberculose e faleceu; a autópsia mostra paracoccidioidomicose. Este caso seria classificado como desacordo tipo 1 , pois o hospital dispõe dos recursos para o dignóstico diferencial, a doença tem terapêutica conhecida e eficiente e o paciente era potencialmente tratável.

A classificação utilizada baseou-se naquelas originariamente propostas por Goldman ${ }^{21}$ e Anderson', sendo que reunimos em um só grupo, definido por nós como desacordo 3 , as classes III e IV. Consideramos ser isso possível já que sob o ponto de vista de prognóstico elas não podem ser completamente separadas, e ambas agrupam diagnósticos de menor importância ou não diretamente relacionados à causa mortis.

"Desacordo 1" - Doença importante que, se diagnosticada clinicamente, alteraria o prognóstico.

Exemplo:

Diagnóstico clínico: rinotraqueobrónquite.

Diagnóstico anatomopatológico: broncopneumonia bilateral.

"Desacordo 2" - Não diagnóstico de doença importante que, se diagnosticada clinicamente, não alteraria o prognóstico.

Exemplo:

Diagnóstico clínico: 1) malformação da drenagem linfática; 2) abscesso pulmonar.

Diagnóstico anatomopatológico: .1) carcinomatose generalizada com linfangite carcinomatosa; 2) carcinoma epidermóide com fistulização esôfago-brônquica e metástase ganglionar.

"Desacordo 3" - Não diagnóstico de doença de menor importância, sem influência ou com influência duvidosa no prognóstico.

\section{Exemplo:}

Diagnóstico clínico: 1) hipertensão arterial malígna; 2) acidente vascular cerebral hemorrágico.

Diagnóstico anatomopatológico: 1) hipertensão arterial malígna; 2) acidente vascular cerebral hemorrágico; 3) doença fibrocística da mama.

Analisaram-se ainda a cor, sexo, idade e período de internação.

Por definição, os grupos apresentando "desacordo 1" e "desacordo 2" não apresentam intersecção de casos, já que no "desacordo 2 " o desenvolver dos acontecimentos em direção ao óbito era inevitável. Um caso era considerado como apresentando "desacordo 3" desde que não apresentasse desacordo 1 ou 2.

Todos os prontuários foram analisados por um médico do último ano de residência do Serviço de Anatomia Patológica, juntamente com dois doutorandos (6: ano), sob supervisão de um clínico experiente. A supervisão constituiu na discussão dos casos em que houve dúvidas na aplicação dos critérios, previamente estabelecidos, na discussão de um número aleatório de casos em que essas dúvidas nâo existiram, assim como na discussão de todos os casos classificados como desacordo tipo 1 .

Usamos o teste ' $t$ "' de Student para diferenças entre as médias. Quando necessário usamos a transformação logarítmica das variáveis para ajuste à normalidade. $\mathrm{O}$ teste do $\mathrm{X}^{2}$ foi usado para testar as diferenças entre proporções. O nível de significância adotado foi $p<0,05$.

\section{RESULTADOS}

Entre os diagnósticos não formulados em vida, e observados na necrópsia (falso negativo), os mais frequentes foram: pielonefrite aguda $(100 \%)$, embolia pulmonar $(87,50 \%)$, aneurisma dissecante da aorta $(66,66 \%)$, broncopneumonia $(58,16 \%)$, neurocisticercose $(50 \%)$ e tuberculose $(46,15 \%)$ (Tabela).

Os diagnósticos formulados em vida que mais freqüentemente não tiveram confirmação pela necrópsia (falso positivo) foram: tuberculose $(69,56 \%)$, paracoccidioidomicose $(57,14 \%)$, septicemia $(53,13 \%)$, doença de Chagas $(44,44 \%)$, embolia pulmonar $(50 \%)$ e diabetes $(45,45 \%)$ (Tabela).

Os diagnósticos clínicos que encontraram mais freqüentemente confirmação anatomopatológica foram: meningite purulenta $(100 \%)$, peritonite $(100 \%)$, aneurisma dissecante da aorta $(100 \%)$, hipertensão arterial malígna 
$(100 \%)$, neurocisticercose $(100 \%)$ e neoplasia maligna $(89,47 \%)$ (Tabela). Em 30 dos 200 casos não se encontrou qualquer dos diagnósticos mencionados na Tabela.

Para as entidades mencionadas na Tabela obteve-se um total de 286 diagnósticos clínicos, sendo 83 falso-positivos (não obtiveram confirmação anatomopatológica) e 335 diagnósticos anatomopatológicos onde 132 não foram observados pela clínica (falso-negativo).

Houve concordância diagnóstica em 97 $(48,50 \%)$ dos 200 casos, sendo os percentuais de "desacordo 1" de 9,50\% (19 casos), "desacordo 2 " de $18 \%$ (36 casos) e "desacordo 3" de $24 \%$ (48 casos).

O grupo de pacientes estudados apresentava uma distribuição bimodal em relação à idade com anti-moda de 10 anos. Os 167 casos acima de 10 anos compreendiam $83 \%$ da amostra, com 46 casos com idade de 60 ou mais anos.

O grupo acima de 10 anos de idade apresentava média etária entre 50,78 anos (grupo concordante) e 48,11 anos ("desacordo 1").

Nos pacientes abaixo de 10 anos de idade, a média etária era entre 0,52 anos ("desacordo 2 ") e 2,36 anos ("desacordo 3"').

Comparando-se as médias etárias de dois grupos, um maior ou igual a 60 anos (78 casos) e outro maior de 10 anos e menor que 60 anos, não encontramos diferença significativa em relação à . classificação diagnóstica (CD) ( $p>0,05)$. Não foi possível comparar as médias entre os grupos com idade menor que 10 anos devido ao pequeno número de casos.

Cento e vinte $(60 \%)$ dos 200 casos eram indivíduos brancos, $47(23,50 \%)$ pardos e 33 $(16,50 \%)$ de negros, sendo do total 121 $(60,50 \%)$ do sexo masculino.

Os testes de associação entre sexo e $C D$ $(p>0,05)$, cor e $C D(p>0,05)$, idade e $C D$ $(p>0,10)$ mostraram-se não significativos.

Cento e vinte e quatro $(62 \%)$ dos 200 pacientes permaneceram internados menos que 3 dias, com média de período de internação variando entre 1,47 dias (grupo concordante) e 1,62 dias ("desacordo 3").

Não houve diferenças de erro entre o grupo com períodos de internação de até 3 dias e aquele com mais de 3 dias ( $p>0,80$ ). Em relação às médias do tempo de internação, no grupo acima de 3 dias, quando comparadas concordantes e "desacordo 1 " $(\mathrm{p}<0,05)$, "desacordo 2 " e "desacordo 1 " $(p<0,05)$, houve diferença estaticamente significativa indicando que o "desacordo 1" associa-se ao menor período de internação nos pacientes que permaneceram por mais de 3 dias internados, diferença esta não encontrada naqueles com menos de 3 dias de internação.

\section{DISCUSSÃO}

Em todo o mundo tem havido queda no número de necrópsias, o que provocou a publicação de numerosos trabalhos que vieram demonstrar sua importância 8,10,13,19,23,26,30,32,37,38,42. No Brasil encontramos situação similar, embora a alta discordância diagnóstica $(51,50 \%)$, também relatada em outros trabalhos $8,11.12,23,31$, $37,38,42$, não justifique essa queda e deveria estimular a prática do procedimento.

Observou-se que os diagnósticos discordantes em $9,5 \%$ implicariam alteração para melhor prognóstico. A freqüência desse achado na lite-

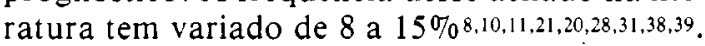

Alguns estudos descreveram maior frequêencia de discrepância diagnóstica em pacientes idosos, justificada na literatura por serem eles frequientemente portadores de mais de uma doença e pela sua própria condição senil8,12,31,37. Em outro estudo ${ }^{18}$ notou-se que essa discrepância, observada no passado, mais recentemente desapareceu, acreditando seus autores que este fato ocorreu devido à introdução de novas técnicas diagnósticas. No presente estudo, os dados mostraram que a freqüência do desacordo diagnóstico não é diferente nos pacientes pediátricos (menores de 10 anos) e nos adultos.

Ao contrário do que se poderia esperar não se notou diferença significativa na freqüência do desacordo diagnóstico entre os grupos com períodos de internação superiores a 3 dias e naqueles com período de internação menor ou igual a 3 dias $^{8}$. Entre pacientes com período de internação superior a 3 dias, os com "desacordo 1" mostraram menor período médio de internação em relação ao dos grupos concordante e "desacordo 2"', indicando que, com menores períodos de internação, o desacordo diagnóstico tende a ser mais grave $8,31,37$. Outro estudo observou $^{9}$, por outro lado, que em períodos de internação acima de um mês aumenta o número de diagnósticos inadequados.

Das entidades nosológicas presentes na Tabela, em média $39 \%\left(132 / 335=\frac{\Sigma(F(-))}{\Sigma \text { DAP }}\right)$ foram somente observadas à necrópsia representando uma quantidade substancial de informação que teria sido perdida caso a necrópsia não tivesse sido realizada. O valor complementar a $39 \%$ pode ser considerado como um indice de sensibilidade de diagnóstico clínico: $61 \%$. Este mostrou-se, no entanto, mais específico como 
representado pela média da estimativa da confirmação anatomopatológica do diagnóstico clínico: $70,97 \%\left(286-83 / 335=\frac{\Sigma \mathrm{DC}-\Sigma(\mathrm{F}(+))}{\Sigma \mathrm{DAP}}\right)$ significando que mesmo quando o diagnóstico clínico possa estar correto há um substancial número de outros diagnósticos de importância que serão revelados somente na necrópsia $8,11,19,23,28,32,38,39$.

Nossos resultados em relação a acidente vascular cerebral, infarto do miocárdio, embolia pulmonar, peritonite, aneurisma dissecante da aorta, broncopneumonias e neoplasia concordaram com os estudos anteriores $8,11,19,23,24,31,32,37,42$.

Os dois grupos de maior dificuldade diagnóstica foram as doenças do aparelho cárdio-respiratório e as doenças de natureza infecciosa ${ }^{11}$. Particularmente estas têm essa dificuldade explicada pela sintomatologia variada, freqüente embricamento de diagnósticos diferenciais e pela dificuldade de isolamento do agente. No presente estudo, a maior percentagem de diagnósticos clínicos não aprovados na necrópsia foi em relação à tuberculose $(69,56 \%)$.

Dos casos de pielonefrite aguda, três $(50 \%)$ tinham acometimento de um rim, sendo em dois deles extenso. $O$ acometimento bilateral ocorreu em dois outros casos, sendo um deles extenso. No sexto caso não foi possivel definir a extensão. Dos casos de acometimento extenso, em um não havia referência de sintomatologia de trato urinário, em outro o paciente (diabético) mostrava sintomas inespecíficos de infecção de trato urinário e sedimento alterado, e no terceiro o paciente apresentou um quadro de septicemia com rápida evolução para o óbito (menos de 24 horas de internação). Muitas são as referências na literatura de que a pielonefrite aguda pode comportar-se assintomaticamente ou de maneira frustra e com sinais e sintomas atípi$\cos ^{6,27,35}$. Nossos resultados confirmaram essas observações e alertam para a dificuldade desse diagnóstico. Para pielonefrite aguda tem-se relatado $82,30 \%$ de diagnóstico anatomopatológico sem suspeita clínica ${ }^{27}$. Os presentes dados mostraram $100 \%$ de diagnósticos somente observados na necrópsia, sendo a provável causa dessa discrepância o pequeno número de casos.

Em relação à paracoccidioiodomicose houve alto índice de diagnósticos clínicos sem confirmação anatomopatológica $(57,14 \%)$, sendo que destes casos $50 \%$ eram portadores de tuberculose. Os diagnósticos diferenciais com tuberculose e neoplasia foram levantados em todos os casos concordantes e naqueles somente descobertos pela necrópsia; no presente material foram problema diagnóstico concordando com o encontrado por outros autores $4,16,36$, podendo estar associadas no mesmo paciente. Encontramos um caso de neoplasia e paracoccidioidomicose concomitantes.

As malformações congênitas concordantes foram atresia do esôfago, meningomielocele, atresia de vias biliares e síndrome de Down. Os dois casos de diagnóstico clínico sem confirmação anatomopatológica resultaram de interpretação errônea, pois um deles, diagnosticado como malformação linfática era, na verdade, uma linfangite carcinomatosa como formação de quilotórax; o outro, diagnosticado como estenose congênita da artéria pulmonar, era uma pancardite reumatismal. Em dois casos o diagnóstico foi somente necroscópico, sendo ambos de malformaçð̃es valculares cerebrais, uma no hipocampo (assintomática) e outra no polígono de Willis, que evoluiu clinicamente com acidente vascular cerebral.

A incidência de neurocisticercose no grupo estudado foi de $3 \%$, confirmando a incidência anteriormente observada em nossa instituição2s. Encontraram-se 3 casos $(50 \%)$ não suspeitados clinicamente, um deles com sintomatologia incontestável. Nos outros dois não foi possível atribuir a sintomologia unicamente à neurocisticercose. Estudos necroscópicos e clínicos têm diferido em seus resultados quanto à presença de casos assintomáticos 3,7,34,41. Alertamos para $o$ alto índice de achados necroscópicos e a relativa frequência da doença.

Em relação à doença de Chagas, notamos também uma grande percentagem de diagnósticos clínicos sem confirmação anatomopatológica. Isso reflete a ausência de formas agudas observadas no presente estudo, a dificuldade de se demonstrar o parasita nas formas crônicas ${ }^{2}$ e o estudo sorológico inadequado, embora em muitos casos o quadro clínico-propedêudico fosse sugestivo.

Algumas doenças são sabidamente de controverso e difícil diagnóstico na necrópsia, dentre as quais, diabetes e septicemia. Apesar disso, a necrópsia demonstrou casos não diagnosticados em $14,28 \%$ e $21,05 \%$, respectivamente. Ainda que haja pouca correlação do diagnóstico clínico com o anatomopatológico, a necrópsia pode mostrar casos com evidência indiscutível de septicemia e diabetes ${ }^{37}$.

Como observações gerais ressaltamos alguns aspectos:

1 - Algumas entidades nosológicas estudadas são em pequeno número, porém os presentes resultados são concordantes com os da literatura.

2 - A necrópsia tem sido freqüentemente considerada como um dos métodos mais se- 
guros da avaliação da hipótese clínica, no entanto, continua pouco solicitada ${ }^{38}$. Muitos clínicos queixam-se de que a necrópsia é realizada de maneira imprópria e não responde aos seus anseios e dúvidas.

3 - Acreditamos que a maneira de se incrementar a relação anatomoclínica, descrita recentemente ${ }^{38}$, consiste na troca constante de idéias entre clínicos e patologistas visando à melhoria na qualidade do serviço de ambos.

Torna-se, pois, evidente a importância da necrópsia como meio de aprimoramento do diag- nóstico clínico e fonte de dados epidemiológicos. Sempre que possível, o preenchimento do atestado de óbito deve ser feito após o conhecimento dos dados necroscópicos 8,10,19,21,23,24,26,29,32.

\section{AGRADECIMENTOS}

Aos Drs. Mário A.P. Moraes, Luciano de Souza Queiroz e Luis Antonio Kannebley Bittencourt, pelas úteis sugestões; ao Dr. Luiz A. Magna, pela colaboração na análise estatística dos dados.

ALMEIDA, M.C. de et al. [Correlation between anatomo-clinical diagnosis and retrospective assessment of clinical diagnosis in post mortms]. Rev. Saúde públ., S. Paulo, 23:285-91, 1989.

ABSTRACT: The post mortem findings in 200 autopsies were compared with the clinical diagnoses. Twenty diseases were analysed with respect to clinico-pathological correlation. In relation to the respective totals the diseases most frequently missed clinically were (false negative) pyelonephrites $(100 \%)$, pulmonary embolus $(87.50 \%)$ and bronchopneumonia $(58.16 \%)$. In relation to the respective totals the clinical diagnoses less frequently confirmed (false positive) were tuberculosis $(69.56 \%)$, paracoccidioidomycosis $(57.14 \%)$, sepsis $(53.13 \%)$ and Chagas'disease $(44.44 \%)$. There was clinicopathological agreement in 97 autopsies $(48.50 \%)$. In 19 cases $(9.50 \%)$ if the diagnostic error had been detected during life this probably would have changes the prognosis. The findings are discussed in the light of previous studies. The importance of routine post-mortem examination and clinico-pathological correlation is stressed.

KEYWORDS: Diagnosis. Autopsy. Diagnostic error. Evaluation.

\section{REFERÊNCIA BIBLIOGRÁFICA}

1. ANDERSON, R.E. The autopsy as an instrument of quality assessment: classification of premortem and postmortem discrepancies. Arch. Pathol. Lab. Med, 108: 490-4, 1984.

2. ANDRADE, Z.A. Anatomia patológica. In: Cançado, J.R., ed. Doença de Chagas. Belo Horizonte. Imprensa Oficial do Estado de Minas Gerais, 1968. p. 315-43.

3. ARROYO-GONZALES, M. ApUd TAKAYANAGUI, O.M. \& JARDIM, E. Aspectos clínicos da neurocisticercose: análise de 500 casos. Arq. Neuropsiquiat., S.Paulo, 41: 50-64, 1983.

4. BARBOSA, W. \& DAHER, R.R.H. Blastomicose sulamericana (Paracoccidioidomicose). In: Veronesi, R. Doenças infecciosas e parasitárias. 7 a ed. Rio de Janeiro, Ed. Guanabara Koogan, 1982. p. 638-51.

5. BATTLE, R.M.; PATHACK, D.; HUMBLE, C.G.; KEY, C.R.; VANATTA, P.R.; ROLLA, B.H.; ANDERSON, R.E. Factors influencing discrepancies between premortem and postmortem diagnoses. J. Amer. med. Ass., 258: 339-44, 1987.

6. BLACKLOW, R.S. MacBryde's signs and symptoms: applied pathologic physiology and clinical interpretation. $6^{\text {th }}$ ed. Philadelphia, J.B. Lippincott, 1983, p. 193-4.

7. BRICEÑO, C.E.; BIAGI, F.; MARTINEZ, B. Apud MÀRQUES-MONTEZ, H. Cysticercosis. In: Maciel Rojas, R.A., ed. Pathology of protozoal and helminthic diseases. New York, Williams and Wilkins, 1971. p. 582-616.

8. BRITTON, M. Diagnostic errors discorvered at autopsy. Acta med. scand., 196: 203-10, 1974.
9. CAMERON, H.M. The autopsy: its role in modern hospital practice. Invest. Cell Pathol., 1: 297-300, 1978.

10. CAMERON, H.M. \& MacGoOGAN, E. A prospective study of 1152 hospital autopsies. I Inaccuracies in death certification. J.Pathol.,133: 273-83, 1981.

11. CAMERON, H.M. \& MacGOOGAN, E. A prospective study of 1152 hospital autopsies. II Analysis of inaccuracies in clinical diagnoses and their significance. J. Pathol., 133: 285-300, 1981.

12. CAMERON, H.M.; LOWE, J.; MacGOOGAN, E.; CARDESA, A. Summary of the symposium on "The Hospital Autopsy: Its Contribution to Medical Audit". Pathol.Res.Pract., 181: 480-1, 1986.

13. COUNCIL ON SCIENTIFIC AFFAIRS. Autopsy: a comprehensive review of current issues. J. Amer. med. Ass., 258: 364-9, 1987.

14. DAHMS, B. The autopsy in pediatrics. Amer. J. Dis. Child., 140: 335, 1986.

15. DEGOWIN, E.L. \& DEGOWIN, R.L Bedside diagnostic examination, $4^{\text {th }}$ ed. New York, Macmillan, 1981.

16. DEL NEGRO, G. Outras lesð̃es: formas de ocorrência rara e associação com outros processos. In: Del Negro, G.; Lacaz, C.S.; Fiorillo, A.M., Ed. Paracoccidioidomicosis: blastomicose Sul-Americana. São Paulo, Sarvier, 1982. p. 229-40.

17. FARIA, J.L. Anatomia patológica. II - Parte especial. Rio de Janeiro, Ed. Guanabara Koogan, 1975. 
18. GIBINSKI, K.; HARTLEB, M.; KOTURBASZ, D. Comparison between pre and post-mortem diagnosis in a consecutive series of patients. Scand. J. Gastroenterol., 20: 370-2, 1985.

19. GOBBATO, F.; VECCHIET, F.; BARBIERATO, D.; MELATO, M.; MANCONI, R. Inaccuracy of death certificate diagnoses in malignancy: an analysis of 1,405 autopsies cases. Hum. Pathol., 13: 1136-8, 1982.

20. GOLDMAN, J. Diagnostic advances Vs the value of autopsy. Arch. Pathol. Lab. Med., 108: 501-5, 1984.

21. GOLDMAN, J.; SYASON, R.; ROBBINS, S.; COHN, L.H.; BETTMANN, M. WEISBERG, $M$. The value of autopsy in three medical eras. New Engl. J. Med., 308: 1000-5, 1983.

22. GOUGH, J. Correlation between clinical and autopsy diagnoses in a community hospital. Canad. med. Ass. J., 133: 420-2, 1985.

23. HARTVEIT, F. Clinical and post-mortem assessment of the cause of death. J. Pathol., 123: 193-210, 1977.

24. HARTVEIT, F. Autopsy findings in cases with a clinically uncertain cancer diagnosis. J. Pathol., 129: 111-9, 1979.

25. HELLMEISTER, C.R. \& FARIA, J.L. Neurocisticercóse: dados necroscópicos. Rev. Ass. med. bras., 19: 281-2, 1973

26. KIRCHER, T.; NELSON, J.; BURDO, H. The autopsy as a measure of accuracy of the death certificate. New Engl. J. Med., 313: 1263-9, 1985.

27. KLEEMAN, C.R.; HEWITT, W.L.; GUZE, L.B. Acute pyelonephritis. Medicine, Baltimore, 39: 57.9, 1960.

28. LANDFELD, C.S.; CHREN, M.-M.; MYERS, A.; GELLER, R.; ROBBINS, S.; GOLDMAN, L. Diagnostic yield of the autopsy in a university hospital and a community hospital. New Engl. J. Med., 318: 1249-54, 1988.

29. MacFARLANE, M.J.; FEINSTEIN, A.R.; WELLS, C.K.; CHAN, C.K. The "epidemiologic necropsy": unexpected detections, demographic selections, and changing rates of lung cancer. J. Amer. med. Ass., 258: 331-8, 1987.

30. MCPHEE, S.J. \& BOTTLES, K. Autopsy: moribund art or vital science? Amer. J. Med., 78: 107-13, 1985.
31. MERCER, J. \& TALBOT, I.C. Clinical diagnosis: a post-mortem assessment of accuracy in the 1980 . Postgrad. Med. J., 61: 713-6, 1985.

32. MOLLO, F.; BERTOLDO, E.; GRANELI, G.; CARVALHO, F. Reliability of death certifications for different types of cancer. An autopsy survey. Pathol. Res. Pratic., 181: 442-7, 1986.

33. NEMETZ, P.N.; LUDWING, J.; KURLAND, L.T. Assesing the autpsy. Amer. J. Pathol., 128: 362-78, 1987.

34. QUEIROZ, A.C. \& MARTINEZ, A.M.B. Envolvimento do sistema nervoso central na cisticercose. Arq. Neuropsiquiatr., S.Paulo, 37: 34-41, 1971.

35. RAMOS Jr., J. Semiotécnica da observação clínica: síndromes clínico-propedêuticas, 6: ed. São Paulo, Ed. Sarveir, 1980. p. 865-7.

36. RIZZON, C.F.C.; SEVERO, L.C.; PORTO, N.S. Paracoccidioidomicose - Estudo de 82 casos observados em Porto Alegre - RS. Rev. Ass. med. $R$. G. Sul, 24:15-7, 1980.

37. SANDRITTER, W.; SATAEUDINGER, M.; DREXLER, H. Autopsy and clinical diagnosis. Pathol. Res. Pratic., 168: 107-14, 1980.

38. SCHNED, A.R.; MOGIELNICKI, P.R.; STAUFFER, M.E. A comprehensive quality assessment program on the autopsy service. Amer. J. clin. Pathol., 86:133-8, 1986.

39. STEVANOVIC, G.; TUCANOVIC, G.; DOTLIC, R.; KANJUH, V. Correlation of clinical diagnoses with autopsy finding: a retrospective study of 2145 autopsies. Hum. Pathol., 17: 1225-30, 1986.

40. SVENDSEN, E. \& HILL, R.B. Autopsy legislation and practice in various contries Arch. Pathol. Lab. Med., 111: 846-50, 1987.

41. TAKAYANAGUI, O.M. \& JARDIM, E. Aspectos clinicos da neurocisticercose: análise de 500 casos. Arq. Neuropsiquiatr., S.Paulo, 41: 50-64, 1983.

42. THURLBECK, W.M. Accuracy of clinical diagnosis in a Canadian teaching hospital. Canad. med. Ass. J., 125: 443-7, 1981.

Recebido para publicação em 20/9/1988. Reapresentado em 5/4/89. Aprovado para publicação em 24/4/89. 